\title{
Computational efficient segmentation of cell nuclei in 2D and 3D fluorescent micrographs
}

\author{
Jonas De Vylder and Wilfried Philips \\ Ghent University - IBBT - Dept. of Telecommunications and Information Processing, \\ St.-Pietersnieuwstraat 41,9000 Ghent, Belgium
}

\begin{abstract}
This paper proposes a new segmentation technique developed for the segmentation of cell nuclei in both 2D and 3D fluorescent micrographs. The proposed method can deal with both blurred edges as with touching nuclei. Using a dual scan line algorithm its both memory as computational efficient, making it interesting for the analysis of images coming from high throughput systems or the analysis of 3D microscopic images. Experiments show good results, i.e. recall of over 0.98 .
\end{abstract}

Keywords: image analysis, segmentation, nuclei detection, computational complexity

\section{INTRODUCTION}

Recent research has led to the development of a wide range of new imaging instruments such as Scanned Light Sheet Microscopy and high-throughput screening. These innovations have led to increasing insight in embryo development, tissue repair, plant growth, etc. ${ }^{1,2}$ These new imaging technique however come with a major bottleneck: they produce huge amounts of data. This data contains valuable information, but has to be interpreted before a biologist is able to use this information. One of the most important objects in micrographs to be used for generic purposes, are cells or nuclei. Due to their importance, several automated detection techniques have been reported in literature.

Two main approaches can be found in these techniques: intensity based and edge based techniques. The first group generally first thresholds the image in order to find cell nuclei. ${ }^{3-8}$ However if nuclei touch, or partially occlude each other, they can't be distinguished and they are considered to be one nucleus. So an extra post processing step is needed to separate these nuclei. This is often done based on shape information, e.g. using watershed segmentation on the distance transform of the thresholded image. This only works if the shape shows clear concavities near the place where the nuclei touch. This unfortunately is not always the case.

The second group mentioned is based on edge information. ${ }^{9-12}$ An example of such a technique is watershed segmentation. This technique has the risk of over segmenting nuclei due to noise, or under segmentation due to a small weakness in the edges, i.e. leaking. Using a region merge strategy the first problem might be solved, ${ }^{7,8}$ but the second problem is more difficult to tackle since it's practically the same as splitting segments in thresholded images. Instead of using the watershed algorithm, an interesting approach is reported in. ${ }^{13}$ They calculate a force field where the forces point towards close by edges. By inversing this force field, they can find the center of a nucleus and by extension, each pixel pointing to the same center, i.e. belonging to the same segment. The force field is calculated in such a way that it is robust to small weaknesses in the edge and robust against noise. The downside of this technique is that it's computational expensive to calculate the force field and even more time consuming to find each pixel corresponding to the same segment. This makes this algorithm impractical to use for large 3D sets or for 2D sequences which require fast computation, e.g. data coming from high through put systems. In this paper we propose a new segmentation technique based on the work of. ${ }^{13}$ This paper will use a new energy field, which is similar to using a force field. This energy field has the same advantages considering robustness, but can be efficiently computed using a dual scan line algorithm. Furthermore are there efficient segmentation techniques available to use with the proposed energy field, whereas segmentation using a force field remains a computational burden.

Corresponding author: Jonas De Vylder

Jonas De Vylder: E-mail: jonas.deVylder@telin.ugent.be, Telephone: +3292643416 
This article is arranged as follows: in the following section we will elaborate on our proposed energy field, how it can be computational efficiently be calculated and how it can be used to detect and segment the nuclei. In section 3 we validate our algorithm both on synthetic as on real 2D data sets. Next, we analyze both in a theoretical as in an experimental way the computational demands of the proposed method. Section 4 recapitulates and concludes.

\section{IMAGE ANALYSIS}

\subsection{Nuclei Detection}

$\mathrm{In}^{10}$ nuclei segmentation is done using a force field. At each pixel a particle is placed. This particle moves due to the force field. A group of particles will cluster at sinks, i.e. places where the force field is zero, or where opposing forces cancel each other. Li et al. ${ }^{13}$ proposes the use of the gradient vector flow (GVF) field ${ }^{14}$ as a force field. The GVF force field is calculated by iteratively updating the external force field in order to minimize a specific energy function. Although this force field has been proven useful, it comes at a great cost: iteratively updating a force field in order to minimize an energy function is both memory and time consuming. A different force field, vector field convolution (VFC) force field ${ }^{10}$ does not suffer from this problem, but it is difficult to find an optimal kernel function for a specific dataset. In the remaining of this section, we propose a new energy based on feature propagation $(f p)$, which does not need the iterative optimization of a force field, but which has straightforward parameters.

We start from a feature map $F_{f p}(x, y)$, e.g. an edge map where the feature map expresses how much evidence there is that there is an edge at pixel $(x, y)$. The goal is to create an energy map, $E_{f p}(x, y)$, where there is high edge evidence at the edge itself and where the edge evidence gradually decreases if you get further away from the edge. The main idea is to propagate strong edge evidence at a certain pixel to its neighboring pixels with lower edge evidence. This step only propagates edge evidence to the direct neighbors, which would require an iterative process as well. This can however be avoided by the following dual scan line algorithm:

1. Scan the feature map row by row from top to bottom

2. In each row, the pixels are scanned from left to right

3. Define the already processed neighborhood of a pixel $(x, y)$, i.e.

$$
\mathbf{n}(x, y, i)= \begin{cases}(x, y-1) & \text { if } i=1 \\ (x-1, y-1) & \text { if } i=2 \\ (x-1, y) & \text { if } i=3 \\ (x-1, y+1) & \text { if } i=4\end{cases}
$$

In Fig. 1(a) the already processed neighbor pixels for $\mathrm{P}$ are shown in pink.

4. Update the pixel in the energy map by:

$$
E_{f p}(x, y)=\max \begin{cases}\max _{i} \gamma E_{f p}(\mathbf{n}(x, y, i)) & \text { (a) } \\ F_{f p}(x, y) & \text { (b) }\end{cases}
$$

where $\gamma \in[0,1]$ is a weighting coefficient, which determines the speed at which feature evidence decreases. This propagates feature evidence of a pixel beyond its direct neighbors in the scanning direction. Note that all pixels of $E_{f p}(\mathbf{n}(x, y,)$.$) are already processed in previous steps, due to the scanning order.$

This algorithm propagates feature evidence from top to bottom and from left to right of the image. Repeat the algorithm in order to propagate edge evidence in the remaining directions, but in opposite scanning direction, i.e. from bottom to top and from right to left. Then the already processed neighborhood of a pixel $(\mathrm{x}, \mathrm{y})$ is:

$$
\mathbf{n}^{\prime}(x, y, i)= \begin{cases}(x, y+1) & \text { if } i=1 \\ (x+1, y+1) & \text { if } i=2 \\ (x+1, y) & \text { if } i=3 \\ (x+1, y-1) & \text { if } i=4\end{cases}
$$




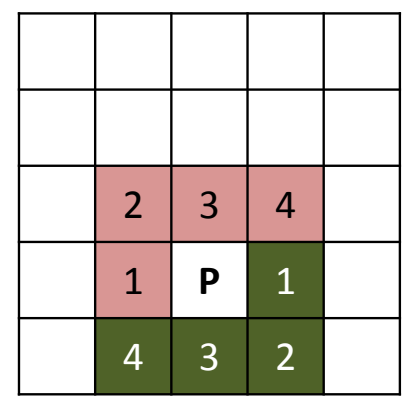

(a)

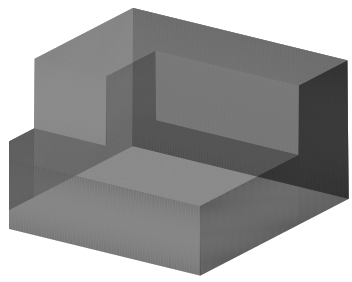

(b)

Figure 1. (a) An example of processed neighborhoods used by 2D feature propagation. The red one is used in the first run, the green neighborhood is used for the second run. (b) The first run neighborhood for 3D segmentation.

This new neighborhood is shown in dark green in Fig. 1(a). In this new feature map, the cell nuclei correspond to local minima. However not all local minima are necessarily nuclei. The false detections, i.e. minima, can however easily be detected by comparing the intensity of the original image at the detection to a predefined threshold. On how to chose the best threshold value, we refer to. ${ }^{4}$

Note that this technique of evidence propagation is easily extended to 3D. The already processed neighborhood would then involve the previous processed slice as well, i.e.

$$
\mathbf{n}(x, y, z, i)=\left\{\begin{array}{ll}
(x, y-1, z) & \text { if } i=1 \\
(x-1, y-1, z) & \text { if } i=2 \\
(x-1, y, z) & \text { if } i=3 \\
(x-1, y+1, z) & \text { if } i=4 \\
(x-1, y-1, z-1) & \text { if } i=5 \\
(x-1, y, z-1) & \text { if } i=6 \\
(x-1, y+1, z-1) & \text { if } i=7 \\
(x, y-1, z-1) & \text { if } i=8 \\
(x, y, z-1) & \text { if } i=9 \\
(x, y+1, z-1) & \text { if } i=10 \\
(x+1, y-11, z-1) & \text { if } i=11 \\
(x+1, y, z-1) & \text { if } i=12 \\
(x+1, y+1, z-1) & \text { if } i=13
\end{array} \quad \mathbf{n}^{\prime}(x, y, z, i)= \begin{cases}(x, y+1, z) & \text { if } i=1 \\
(x+1, y+1, z) & \text { if } i=2 \\
(x+1, y, z) & \text { if } i=3 \\
(x+1, y-1, z) & \text { if } i=4 \\
(x-1, y-1, z+1) & \text { if } i=5 \\
(x-1, y, z+1) & \text { if } i=6 \\
(x-1, y+1, z+1) & \text { if } i=7 \\
(x, y-1, z+1) & \text { if } i=8 \\
(x, y, z+1) & \text { if } i=9 \\
(x, y+1, z+1) & \text { if } i=10 \\
(x+1, y-11, z+1) & \text { if } i=11 \\
(x+1, y, z+1) & \text { if } i=12 \\
(x+1, y+1, z+1) & \text { if } i=13\end{cases}\right.
$$

An illustration of such a 3D neighborhood is shown in Fig.1(b). It might be necessary to adjust the algorithm in order to cope with the significant difference between the resolution in the focal plane and the resolution in the z-axis. This can easily be achieved by using different weighting parameters $\gamma_{i}$ in eq. (2), depending if the neighboring pixel $i$ is in the same plane or in the previous processed plane.

\subsection{Nuclei Segmentation}

$\operatorname{In}^{13}$ they use a force field, instead of an energy field. It is possible to efficiently calculate a force field out of the proposed energy field. ${ }^{15}$ This energy field however allows us to efficiently segment the nuclei immediately, without the need of a force field. The proposed energy map has the property that feature evidence gradually decreases when getting further away of the evidence. This has the advantage that if there is a small place where the evidence is less pronounced, it is strengthened by it neighboring evidence. Furthermore is the influence of noise reduced in the vicinity of feature evidence, since this is generally stronger than noise. These where actually the two main causes of problems occurring with the watershed segmentation, which means that the watershed algorithm can be used to segment the propagated feature map. Computational efficient, i.e. processing time and memory allocation efficient watershed algorithms have been reported in. ${ }^{16,17}$ 


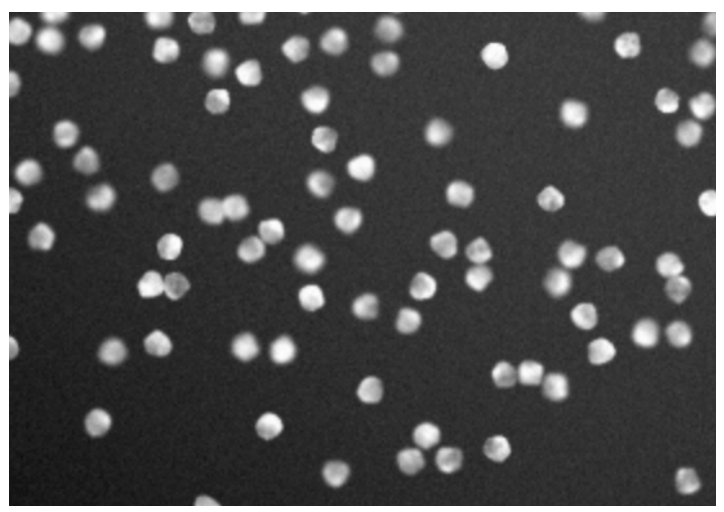

(a) Simulated micrograph

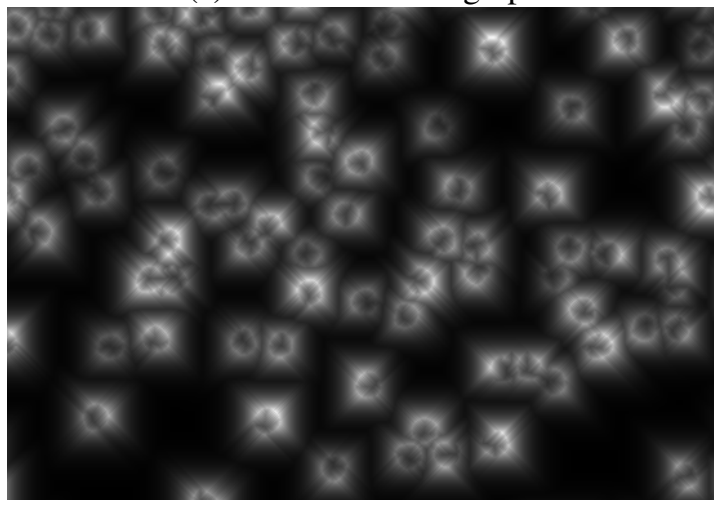

(c) Propagated edge map Detected Nuclei
Figure 2. An example of a simulated micrograph and the detection of cell nuclei in this micrograph.

Although the proposed feature propagation is robust to noise there is still a risk of over segmentation. Mainly with elongated elliptical nuclei, over segmentation might happen, this can however easily be solved using region merging. For each segment it is calculated how much it would cost to merge the segment with a neighboring segment. As a cost function, we choose the minimum value of the feature map along the border between both segments. Then all segments which can be merged without trespassing a certain cost value are merged. Both the calculation of the merging costs between segments as the merging itself can efficiently done using the implementations proposed in. ${ }^{18}$

\section{RESULTS}

\subsection{Nuclei Detection}

As a first validation of the proposed method, a synthetic dataset is analyzed. ${ }^{19}$ These synthetic images were proposed as a common benchmark for nuclei segmentation. The synthetic images show the same intrinsic properties that real microscopic images of cell nuclei would have, e.g. blurred nuclei, non uniform intensity in a nucleus, touching nuclei, non uniform background, etc. In Fig. 3.1(a) an example of such a synthetic image is shown. In Fig. 3.1(b) an edge map is shown. As in realistic images, are not all nuclei contours closed in the edge map and are some edges more clear than others. In Fig. 3.1(c) the energy map, i.e. the propagated edge map can be seen. Finally the result can be seen in Fig. 3.1(d). As can be seen are both isolated as clustered nuclei correctly detected.

A second experiment was done using real 2D data. This data was captured using the high content screen acquisition system ScanR from Olympus. Four images where manually annotated by the author using MTrackJ and imageJ. These images have a resolution of $1344 \times 1024$ pixels and contain on average 153 cell nuclei.

The results of nuclei detection in both synthetic images as in real images are summarized in Table 3.1. The first two rows correspond to the ground truth, i.e. respectively the number of images in the dataset and the number of nuclei shown in the dataset. The next two rows correspond with the number of nuclei detected by the proposed algorithm, i.e. respectively 


\begin{tabular}{|l|rr|}
\hline & synthetic images & real 2D dataset \\
\hline \# images & 20 & 4 \\
\# nuclei & 6000 & 612 \\
\hline total count & 6006 & 643 \\
max error & 3 & 11 \\
\hline precision & 0.996 & 0.933 \\
recall & 0.996 & 0.982 \\
\hline
\end{tabular}

Table 1. Results for the detection of nuclei in both simulated images as in real data.

the number of nuclei counted in the full datasets and the maximum difference between the amount of nuclei visible in the image and the amount of nuclei found in the image. The last two rows correspond to the precision and recall of the full dataset, i.e.

- True positives (TP): each nucleus in the ground truth image which is detected using the proposed algorithm

- False positives (FP): all detections which have no corresponding ground truth nucleus are considered to be FPs

- False negatives (FN): All ground truth nuclei with no corresponding detections are FN's

- Precision is the ratio of the number of TP's in an image, over the total number of detected cells in that image, i.e. including FP's

- Recall is the ratio of the number of TP's in an image, over the total number of cells in the ground truth image

\subsection{Computational Cost}

The proposed method was based on the method defined in. ${ }^{13}$ This method consists of two steps: calculating a force/energy field and then segmenting the image based on this force/energy field. Li et al. proposes the use of the gradient vector flow (GVF) field. The calculation of the GVF field needs $O\left(N^{2}\right)$ operations for each iteration and $N$ iterations are generally needed to calculate the force field. ${ }^{10}$ So the GVF field has an $O\left(N^{3}\right)$ complexity for a square image with dimension $N \times N$. A different, computational less demanding force field was proposed in, ${ }^{10}$ where they propose vector field convolution (VFC). The VFC force computation has a complexity of $O\left(N^{2} \log N^{2}\right)$ which is determined by the complexity of the 2D FFT and IFFT algorithms used. Our proposed scanning algorithm recalculates the value of each pixel twice, resulting in an $O\left(N^{2}\right)$ algorithm. In Fig. 3 the computation time of GVF, VFC and the proposed force field are compared in function of the image size. Note that the time axis is log scaled. These experimental results were calculated on a computer with an Intel core $171.60 \mathrm{GHz}$ CPU and 4 GB RAM. All algorithms were programmed in C. The GVF code was provided by $\mathrm{Xu}$ and Prince. ${ }^{14}$ The code for VFC was provided by the Virginia Image \& Video Analysis group. ${ }^{10}$ In agreement with the theoretical complexity analysis, the GVF field is the slowest to calculate. The VFC field is much faster than GVF, but is significantly slower than the proposed method. The proposed method has not only a low processing time, but is also efficient considering memory usage. Only the current slide and the previous row/slice should be stored in memory.

As already mentioned does this framework exists of two steps. In the second step, the nuclei are effectively segmented using the calculated force/energy field. In, ${ }^{10}$ this is done by following the force field towards sinks. This has to be done for each pixel, meaning that in the worst case the algorithm is $O\left(N^{4}\right)$. In this paper we have proposed to use an energy field instead of a force field. By doing so, we can find nuclei easily, since they correspond with local minima. These can easily be found using a watershed algorithm. There exist memory and computational efficient implementations of the watershed algorithm, resulting in a computational complexity of $O\left(N^{2}\right) \cdot{ }^{17}$

Due to both memory as processing efficient implementation, the proposed method can be used on a standard home computer in contrast to a computer grid of 1000 computers, such as mentioned in. ${ }^{1}$ 


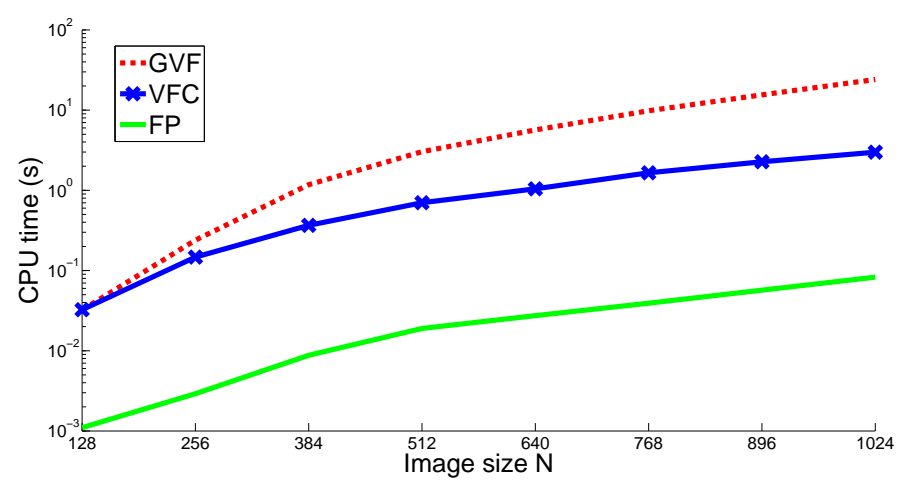

Figure 3. Computational cost of GVF, VFC and feature propagation for a $\mathrm{NxN}$ image.

\section{CONCLUSION}

In this paper a new segmentation algorithm was proposed. The proposed algorithm is developed for the segmentation of cell nuclei and can cope with touching nuclei, even if the surrounding contours don't show clear concavities. The proposed method is calculated using an efficient dual scan line algorithm, making it significantly faster than current state of the art, and only requires to keep two slices in memory. This makes the proposed technique interesting for the analysis of big datasets, such as 3D datasets, or image sequences coming from high throughput systems. The method was tested both on synthetic as on real datasets, resulting in good precision and recall. Precision might be further improved if the found segments would be merged using some form of shape constraints. This is something the authors intend to investigate in the future.

\section{ACKNOWLEDGMENTS}

This research has been made possible by the Institute for the Promotion of Innovation by Science and Technology in Flanders (IWT). We would like to thank the researchers who were willing to make their code or data publicly available, they made a real contribution to the validation of the proposed work. Specifically we would like to acknowledge: Xu, C.; Prince, J.; Li, B.; Acton, S; Ruusuvuori, P.; Lehmussola, A.; Selinummi, J.; Rajala, T.; Huttunen, H. and Yli-Harja, O.

\section{REFERENCES}

[1] Keller, P. J., Schmidt, A. D., Wittbrodt, J., and Stelzer, E. H. K., "Reconstruction of zebrafish early embryonic development by scanned light sheet microscopy," Science 322(5904), 1065-1069 (2008).

[2] Keller, P. J., Schmidt, A. D., Santella, A., Khairy, K., Bao, Z. R., Wittbrodt, J., and Stelzer, E. H. K., "Fast, highcontrast imaging of animal development with scanned light sheet-based structured-illumination microscopy," Nature Methods 7(8), 637-U55 (2010).

[3] Otsu, N., "Threshold selection method from gray-level histograms," IEEE Transactions on Systems Man and Cybernetics 9(1), 62-66 (1979).

[4] Sezgin, M. and Sankur, B., "Survey over image thresholding techniques and quantitative performance evaluation," Journal of Electronic Imaging 13(1), 146-168 (2004).

[5] Cloppet, F. and Boucher, A., "Segmentation of complex nucleus configurations in biological images," Pattern Recognition Letters In Press, Corrected Proof (2010).

[6] Wright Cell Imaging Facility, Manual for the WCIF-ImageJ collection (2010). http://www. uhnresearch. ca/facilities/wcif/imagej/particle\_analysis.htm.

[7] Chen, X. W., Zhou, X. B., and Wong, S. T. C., "Automated segmentation, classification, and tracking of cancer cell nuclei in time-lapse microscopy," IEEE Transactions on Biomedical Engineering 53(4), 762-766 (2006). 
[8] Carpenter, A. E., Jones, T. R., Lamprecht, M. R., Clarke, C., Kang, I. H., Friman, O., Guertin, D. A., Chang, J. H., Lindquist, R. A., Moffat, J., Golland, P., and Sabatini, D. M., "Cellprofiler: image analysis software for identifying and quantifying cell phenotypes," Genome Biology 7(10), - (2006).

[9] Vincent, L. and Soille, P., "Watersheds in digital spaces: An efficient algorithm based on immersion simulations," IEEE PAMI, 1991 13(6), 583-598 (1991).

[10] Li, B. and Acton, S., "Active contour external force using vector field convolution for image segmentation," IEEE transactions on image processing 16, 2096-2106 (2007). http://viva.ee.virginia.edu/research_ vfC.html.

[11] Vincent, L., "Morphological grayscale reconstruction in image analysis: application and efficient algorithms," IEEE transactions on image processing 2, 176-201 (1993).

[12] De Vylder, J., Rooms, F., and Philips, W., "Segmentation of cell nuclei in arabidopsis thaliana roots," in [Proc. of the 7th Int. Conf. on Image Analysis and Recognition (ICIAR2010)], Lecture Notes in Computer Science, Springer Verlag, Pvoa de Varzim, Portugal (June 2010).

[13] Li, G., Liu, T., Nie, J., Guo, L., Chen, J., Zhu, J., Xia, W., Mara, A., Holley, S., and Wong, S. T. C., "Segmentation of touching cell nuclei using gradient flow tracking," Journal of Microscopy-Oxford 231(1), 47-58 (2008).

[14] Xu, C. and Prince, J., "Snakes, shapes and gradient vector flow," IEEE transactions on image processing 7, 359-369 (1998). http://iacl.ece.jhu.edu/projects/gvf/.

[15] De Vylder, J. and Philips, W., "A computational efficient external energy for active contour segmentation using edge propagation," in [Proc. of the IEEE Int. Conf. Image Processing (ICIP2010)], (September 2010).

[16] De Smet, P. and Pires, R., "Implementation and analysis of an optimized rainfalling watershed algorithm," in [proc. of Image and Video Communications and Processing 2000], SPIE (2000).

[17] De Bock, J. and Philips, W., "Line segment based watershed segmentation," in [Advances in Computer Vision and Computer Graphics, Third International Conference on Computer Vision/Computer Graphics], Lecture Notes in Computer Science, 579-586 (2007).

[18] De Bock, J., Pires, R., De Smet, P., and Philips, W., “A fast dynamic border linking algorithm for region merging," in [Advanced Concepts for Intelligent Vision Systems, ACIVS, 8th international conference], Lecture Notes in Computer Science, 232-241 (2006).

[19] Ruusuvuori, P., Lehmussola, A., Selinummi, J., Rajala, T., Huttunen, H., and Yli-Harja, O., "set of synthetic images for validating cell image analysis," in [Proc. of the 16th European Signal Processing Conference (EUSIPCO-2008)], (2008). 